\title{
Peer Instruction no aprendizado da montagem de articulador semiajustável
}

\author{
Ana Rosa Victor dos Santos*; Jessica de Oliveira Vianna Lucas**; Aurimar de Oliveira Andrade***; \\ Armando Hayassy****; Marceli França*****; Fernanda Nunes de Souza*** \\ * Graduanda em Odontologia, Faculdades São José \\ ** Especialista em Odontopediatria, Universidade Federal \\ Fluminense \\ *** Docente, Faculdades São José \\ **** Diretor da Faculdade de Odontologia, Faculdades São José \\ ***** Especialista em Prótese Dentária
}

Recebido em 13/03/2018. Aprovado em 17/02/2019.

\begin{abstract}
RESUMO
As metodologias ativas de ensino-aprendizagem são um desafio tanto para o docente quanto para o discente e instituições de ensino. O presente estudo objetivou diagnosticar as condições mais favoráveis ao aprendizado, comparando diferentes métodos de ensino sobre montagem de articulador semiajustável (ASA) para estudantes de terceiro período da Faculdade de Odontologia São José, Rio de Janeiro, Brasil. Participaram deste estudo 140 estudantes divididos em dois grupos, Grupo I: Leitura de artigo e aula expositiva e Grupo II: Peer Instruction (PI). Foram avaliados o nível de participação nas atividades propostas, a forma como o conhecimento era construído, o número de erros e acertos obtidos nas atividades práticas, além do domínio do assunto abordado e a postura profissional do aluno. Observou-se que no Grupo I $6(21,4 \%)$ estudantes realizaram correta montagem dos modelos e no Grupo II 26 (29,2\%) apresentaram este resultado. Os erros mais prevalentes ocorreram nas fases compensação de cera (Grupo I: 18 - 35,3\%; Grupo II: 26 - 29,2\%) e registro intermaxilar (12 - 23,5\% no Grupo I; 17 - 19,1\% no Grupo II). O resultado obtido comprovou que a proposta de ensino PI, empregada no Grupo II, favoreceu o aprendizado, uma vez que houve aumento do número de acertos nas atividades e domínio dos conceitos que envolvem o manuseio e a operação dos equipamentos utilizados.

Descritores: Educação Superior. Oclusão Dentária. Aprendizagem Ativa.
\end{abstract}




\section{INTRODUÇÃO}

O ensino de Odontologia no Brasil tem apresentado diversas mudanças, dentre as quais a contínua introdução de novas tecnologias de ensino, determinando que novas pesquisas científicas sobre sua eficácia sejam realizadas. Tais tecnologias de informação e comunicação inseridas no campo educacional estão proporcionando novos debates em torno do conhecimento ${ }^{1}$.

A aquisição da destreza, conhecimento, processos afetivos e valores profissionais definem a competência prática na Odontologia, para tanto, a regulação e a avaliação dos métodos de aprendizado tornam-se componentes essenciais da educação, úteis tanto para a melhoria na qualidade do ensino como para a qualidade dos egressos ${ }^{2,3}$.

Para a formação de profissionais da saúde alguns estudos apontam que o modelo tradicional (clássico ou cartesiano) que vem há décadas sendo utilizado é insuficiente em face de nova realidade ${ }^{4}$. Desconsidera as particularidades do Sistema Único de Saúde (SUS) e ignora a insuficiência de profissionais preparados para lidar com as demandas da realidade brasileira, uma vez que precisam ter formação continuada para alcançar um perfil crítico-reflexivo e ter capacidade de desenvolver trabalhos em grupo ${ }^{5,6}$.

As metodologias ativas (MA) são uma concepção educativa que estimulam processos de ensino-aprendizagem crítico-reflexivos, nos quais o educando participa e se compromete com seu aprendizado, propiciando rendimento satisfatório e a reflexão do discente sobre os problemas. As MA disponibilizam recursos para pesquisar problemas e soluções; identificam e organizam as soluções hipotéticas mais adequadas à situação e a aplicação das soluções ${ }^{4}$.

A insatisfação de universitários com as práticas educativas e, em especial, com o distanciamento entre a teoria e a prática, é evidente no exercício da profissão ${ }^{7}$. Segundo algumas pesquisas, tal aspecto é frequentemente apontado como fator principal para a evasão nos cursos de graduação em Odontologia ${ }^{8-11}$ e determinante na escolha profissional ${ }^{12-16}$.

Com o propósito de reduzir a evasão e atenuar o grau de desalento vivenciado pelos alunos, as MA podem auxiliar o desenvolvimento cognitivo do educando frente aos desafios da profissão escolhida. Nessa perspectiva, o Peer Instruction ou Instrução pelos colegas (IpC) é um método ativo de aprendizagem que pode amparar a difícil tarefa de elucidar linguagens teóricas, apropriar as demandas cotidianas da profissão. $\mathrm{O}$ método se destacou por utilizar uma abordagem por meio da qual os estudantes são dispostos enquanto protagonistas da aprendizagem, de tal maneira que o conhecimento proporcione ao educando habilidades e competências que o auxilie na solução de problemas cotidianos ${ }^{17-20}$.

As MA estão alicerçadas na autonomia, um princípio teórico significativo explícito na invocação de Paulo Freire ${ }^{21}$. Nessa lógica, a aprendizagem deve envolver participação ativa do discente, por meio da escrita, leitura, discussão, resolução de problemas e construção de uma síntese e análise. Por isso, tal metodologia tem sido utilizada em diferentes áreas do conhecimento $^{22-25}$.

O presente estudo tem como objetivo avaliar a MA IpC na disciplina de oclusão do Curso de Odontologia das Faculdades São José, Rio de Janeiro, Brasil, comparando-a com abordagem tradicional (aula expositiva, leitura e discussão prévia de artigo sobre o tema).

\section{METODOLOGIA}

A pesquisa foi aprovada pelo comitê de ética em pesquisa institucional (CAAE 55725416. 0.0000.5259). Foi desenvolvido um estudo observacional no período de um ano letivo. Participaram deste estudo 140 estudantes que foram aleatoriamente divididos em dois grupos: 
Grupo I ( $\mathrm{n}=51)$, leitura prévia de artigo sobre o tema proposto e aula expositiva com discussão; Grupo II ( $\mathrm{n}=89)$, método IpC. Foi avaliado o nível de participação nas atividades propostas, a forma como o conhecimento era construído, o número de acertos obtidos nas atividades práticas, além do domínio do assunto abordado e a postura profissional do estudante.

Os estudantes do Grupo I, na primeira etapa, a dos conceitos, receberam um artigo sobre os principais passos da montagem em articulador. Estes conceitos foram abordados sob a forma de leitura e debate em sala de aula, onde foram levantadas as dúvidas e respondidas perguntas sobre o artigo em forma de teste.

No Grupo II, o grupo do método IpC, os estudantes igualmente receberam o mesmo artigo $\mathrm{e}$, adicionalmente, foi enviado por e-mail um teste para comprovar a leitura do artigo. As dúvidas foram discutidas em sala de aula, quando o professor fez uma exposição inicial de um conceito ou conteúdo por não mais que vinte minutos, quando então apresentou um teste conceitual de múltipla escolha a ser respondido individualmente pelos alunos (aproximadamente dois minutos $)^{17}$. As respostas foram informadas ao docente por cartelas coloridas (flashcards) com cor correspondente à alternativa escolhida.

Caso a frequência de acertos se situasse entre $30 \%$ e $70 \%$, os estudantes eram orientados a formar pequenos grupos, preferencialmente com colegas que optaram por alternativas (cores) diferentes no teste conceitual, e discutir por cerca de três minutos, quando votavam novamente. $\mathrm{O}$ objetivo foi que refletissem individualmente e, depois, discutissem em grupo suas respostas, antes de saber qual era a correta.

Conforme as respostas e o número de acertos o docente prosseguia com a explicação ou dava seguimento com a discussão em pares e após a discussão realiza novamente as perguntas. Se o número de acertos fosse maior que $70 \%$, passava para o item seguinte. Caso houvesse significativa porcentagem de erros e o número de acertos fosse menor que $30 \%$ a aula era interrompida e os estudantes reviam os conceitos $^{20}$.

\section{RESULTADOS}

Para a maioria dos estudantes (132-94,3\%) este foi o primeiro contato com o procedimento proposto, os demais já haviam realizado cursos técnicos na área, como, técnico em prótese dentária, em higiene bucal ou tiveram experiência em clínicas ou consultórios odontológicos.

Entre os participantes do estudo 105 (75\%) eram mulheres e 35 (25\%) homens. Na tabela 1 são descritos os erros por etapa e grupo, assim como por gênero. Observou-se que no Grupo I 6 $(21,4 \%)$ estudantes realizaram correta montagem dos modelos e no Grupo II 26 (29,2\%) alunos apresentaram este resultado. Os erros mais prevalentes nos grupos ocorreram na fase compensação de cera (Grupo I 18 - 35,3\% e Grupo II 26 - 29,2\%), no registro intermaxilar (12 - 23,5\% no Grupo I e 17 - 19,1\% no Grupo II). Deve se ressaltar que apesar do número de acertos no Grupo II ter sido maior que no I, 14 (27,5\%) do Grupo I e 14 (15,7\%) do Grupo II não concluíram as atividades propostas por não entregar o relatório final das atividades.

\section{DISCUSSÃO}

O presente estudo avaliou o efeito do método ativo IpC comparado à leitura e discussão de artigo sobre o tema no desempenho dos estudantes do terceiro período da graduação de Odontologia na aplicação dos conceitos teóricos sobre montagem de modelos de estudo em ASA, bem como na aplicação dos conceitos de oclusão na análise dos mesmos.

Quanto à diferença de desempenho entre os gêneros, a proporção de montagem correta no Grupo I foi de $25 \%$ para homens e $5,7 \%$ para mulheres, e no Grupo II de 36,8\% para homens e 
27,1\% para mulheres. É possível se observar que apesar da literatura não ser consistente em relação à diferença de gênero quanto a exploração de temas propostos ${ }^{3}$, em ambos os gêneros houve o aumento na porcentagem de acerto e alcance dos objetivos da disciplina no Grupo II.

Tabela 1. Erros cometidos pelos grupos em estudo, por etapa da atividade e por gênero

\begin{tabular}{|c|c|c|c|c|c|c|c|c|}
\hline \multirow[b]{2}{*}{ Grupo } & \multirow[b]{2}{*}{ Gênero } & \multicolumn{5}{|c|}{ Erros na Montagem (número de alunos) } & \multirow[b]{2}{*}{$\begin{array}{c}\text { Montagem } \\
\text { Correta } \\
\text { n - \% }\end{array}$} & \multirow[b]{2}{*}{$\begin{array}{c}\text { Sem } \\
\text { Relatório } \\
\text { n - \% }\end{array}$} \\
\hline & & $\begin{array}{l}\text { Arco } \\
\text { facial } \\
n-\%\end{array}$ & $\begin{array}{c}\text { Modelo } \\
\text { Superior } \\
\text { n - \% }\end{array}$ & $\begin{array}{c}\text { Registro } \\
\text { Intermaxilar } \\
\mathbf{n}-\%\end{array}$ & $\begin{array}{c}\text { Compensação } \\
\text { Cera } \\
\text { n - \% }\end{array}$ & $\begin{array}{c}\text { Modelo } \\
\text { Inferior } \\
\text { n - \% }\end{array}$ & & \\
\hline \multirow[t]{2}{*}{ I } & Fem & $0-0$ & $0-0$ & $9-17,6$ & $14-27,5$ & $1-2$ & $2-3,9$ & $9-17,6$ \\
\hline & Masc & $0-0$ & $0-0$ & $3-5,9$ & $4-7,8$ & $0-0$ & $4-7,8$ & $5-9,8$ \\
\hline Total & 51 & $0-0$ & $0-0$ & $12-23,5$ & $18-35,3$ & $1-2$ & $6-11,8$ & $14-27,5$ \\
\hline \multirow{2}{*}{ II } & Fem & $2-2,2$ & $1-1,1$ & $15-16,9$ & $21-23,6$ & $1-1,1$ & $19-21,3$ & $11-12,4$ \\
\hline & Masc & $1-1,1$ & $0-0$ & $2-2,2$ & $5-5,6$ & $1-1,1$ & $7-7,9$ & $3-3,4$ \\
\hline Total & 89 & $3-3,4$ & $1-1,1$ & $17-19,1$ & $26-29,2$ & $2-2,2$ & $26-29,2$ & $14-15,7$ \\
\hline
\end{tabular}

Prado et $a l . .^{5}$ alertam que o modelo tradicional desconsidera as necessidades do SUS e forma profissionais despreparados para lidar com a sua realidade, sem adquirir um perfil crítico-reflexivo e sem capacidades de desenvolver trabalho em grupo. A fim de proporcionar um atendimento mais humanizado à população e a aplicação da atividade profissional baseada em evidências, espera-se que o processo de ensino-aprendizagem possa ser ativo e independente, levando a superação da distância entre teoria e prática, valorizando o trabalho entre o serviço de saúde e a comunidade, sendo articulados ${ }^{22}$.

Neste contexto a participação dos estudantes é um aspecto importante, os resultados revelam que 14 alunos em cada grupo não concluíram as atividades propostas, pois não entregaram o relatório final das atividades. A porcentagem de alunos sem relatório foi menor $(27,5 \%)$ no Grupo I, caracterizando maior participação no grupo II, entretanto fica claro que este aspecto ainda precisa de mais atenção, demonstrando que apesar do PI aumentar a participação em sala de aula e em atividades práticas, ainda são necessárias atribuições de notas à entrega das atividades escritas.

As MA são um desafio para os educadores, visto que para sua utilização não é necessário apenas conhecer os modos de operacionalização, mas também os princípios da pedagogia crítica que delega ao docente o papel de mediador no processo de ensinoaprendizagem, tendo função de conduzir os discentes à observação da realidade e apreensão do conteúdo que dela extraem a partir dos temas apresentados $^{4-6}$.

Sobral e Campos ${ }^{4}$ (2012) enfatizam que se faz necessário alertar que as MA não são um mero conjunto de ferramentas voltadas para a formação profissional ou para ações educativas no serviço de saúde. Essa metodologia exige uma atividade pedagógica pautada em objetivos bem definidos, considerando suas principais características: o sujeito participa ativamente do processo de ensino-aprendizagem, busca o saber e se apropria do conhecimento, reflete 
criticamente sobre o que apreendeu para depois realizar ações e transformar a realidade em que vive.

Crouch et $a l .{ }^{19}$ afirmam que é fundamental para o sucesso do método IpC escolher testes conceituais com níveis de dificuldade adequados. As questões selecionadas, segundo as dificuldades dos alunos, devem abordar um único conceito relevante. Além disso, não devem testar a memória.

Além do aumento do número de acertos nas atividades propostas na disciplina foi notável a maior busca dos alunos já aprovados na disciplina por projetos de iniciação à pesquisa e monitoria, assim como por projetos associados à disciplina, como a Liga Acadêmica de Disfunção Temporomandibular em parceria com o curso de Fisioterapia das Faculdades São José. Entretanto, ainda é notável entre os alunos a preocupação excessiva com avaliação ao invés da valorização da aquisição do conhecimento e do domínio da técnica ${ }^{3}$. Neste sentido, é necessário utilizar metodologias que percebam a prática profissional diante da realidade das pessoas que utilizam o serviço de saúde e não apenas disciplinas que se caracterizam de forma exagerada e volumosa de conteúdo a ser decorado e posteriormente esquecido ${ }^{24}$.

A exigência de maturidade e postura profissional adequada à realidade se confirma nas observações de Super $^{12}$, que utilizou a maturidade para avaliar o estágio de desenvolvimento de carreira alcançado por estudantes de diferentes idades e níveis de instrução frente à aptidão para a tomada de decisões. As habilidades, competências e atitudes de enfrentamento podem não variar com a idade em função da adaptabilidade de carreira, indicando equilíbrio entre o mundo do trabalho e o espaço pessoal, além de habilidades para buscar e aceitar mudanças nos papéis de carreira ao longo do tempo ${ }^{12,13}$.

As dimensões da maturidade propostas por Super ${ }^{12,13}$ seriam: a) capacidade de planejamento, estando relacionada também à autoestima; b) capacidade de exploração; c) informação sobre o mundo do trabalho e as opções oferecidas; d) tomada de decisão, habilidade decorrente da avaliação das possibilidades; e e) orientação à realidade.

O conjunto destas características compõe a prontidão para a tomada de decisão ou a condição do indivíduo para a necessária mudança de comportamento em resposta às demandas destacadas por diversos estudos ${ }^{7-15}$. Fica clara a contribuição das MA para o estímulo e desenvolvimento de tal comportamento.

Apesar do aumento no número de acertos nas atividades, ainda permanece uma concentração de erros nas etapas de registro intermaxilar e compensação de cera. Tais resultados sugerem a necessidade de material didático específico para $\mathrm{o}$ entendimento $\mathrm{e}$ reprodução das relações intermaxilares, dirigido aos alunos em transição do ciclo teórico para o ciclo prático e implantação de um ensino voltado para a autoaprendizagem, aprender a aprender e busca autônoma do conhecimento para a resolução dos problemas concretos encontrados nas atividades diárias.

Desta forma, o uso das MA exige determinados recursos, delegando às instituições de Ensino superior preparar os instrumentos necessários à sua aplicação e juntamente aos docentes e discentes promover uma cultura de responsabilidade, ou seja, envolver a comunidade acadêmica dentro dos princípios da metodologia. Assim, há que conscientizar alunos e professores quanto à responsabilidade de ensinar o conteúdo teórico e cultivar nos discentes o desenvolvimento da reflexão baseado nos resultados das pesquisas 
científicas $^{3}$.

\section{CONCLUSÃO}

O método de ensino PI aplicado na disciplina de oclusão propiciou maior apropriação dos conteúdos teóricos trabalhados em sala de aula, uma vez que foi significativo o aumento dos acertos nos procedimentos técnicos avaliados, evidenciando maior domínio dos conceitos que envolvem o manuseio e a operação dos equipamentos utilizados na disciplina.

\section{ABSTRACT \\ Peer Instruction in learning to mount a semi- adjustable articulator}

The active teaching-learning methodologies are challenging to both the professor and student, and to teaching institutions. The aim of the present study was to diagnose the conditions most favorable to learning, by comparing different methods of teaching third semester students about mounting a Semi-Adjustable Articulator, at the São José Dental School, Rio de Janeiro, Brazil. The participants in this study were 140 students, divided into two groups, Group I: Reading the article, and a lecture demonstration, and Group II: Peer Instruction (PI). The following aspects were evaluated: the level of participation in the proposed activities, the way in which knowledge was constructed, the number of errors and correct outcomes obtained in the practical activities, in addition to command of the subject approached and the student's professional posture. In Group I, 6 (21.4\%) students were observed to perform correct mounting of the models, and in Group II, 26 (29.2\%) presented this result. The most prevalent errors occurred in the stages of compensation with wax (Group I: 18 - 35.3\%; Group II: 26 - 29.2\%) intermaxillary register $(12-23.5 \%$ in Group I; 17 $19.1 \%$ in Group II). The result obtained proved that the PI teaching proposal used in Group II favored learning, because there was an increase in the number of correct outcomes obtained in the activities, and command of the concepts that involved the handling and operation of the equipment used.

Descriptors: Higher Education. Dental Occlusion. Active Learning.

\section{REFERENCIAS}

1. Xavier LN, Oliveira GL, Gomes AA, Machado MFAS, Eloia SMC. Analisando as metodologias ativas na formação dos profissionais de saúde: uma revisão integrativa SANARE. 2014; 13(1):76-83.

2. Souza FN. Análise in vivo da adaptação de apoios em prótese parcial removível. Int J Sci Dent. 2012;2(36):10-7.

3. Souza FN, Barros RN, Almeida CC, França M, Hayassy A. Comparação dos métodos tradicional e ativo de educação no aprendizado de um tema de Oclusão Dentária. Rev ABENO. 2015,15(4):60-6

4. Sobral FR, Campos CJG. The use of active methodology in nursing care and teaching in national productions: an integrative review. Rev Esc Enferm USP. 2012; 46(1):208-18.

5. Prado ML et al. Arco de Charles Maguerez: refletindo estratégias de metodologia ativa na formação de profissionais de saúde. Esc Anna Nery Rev Enferm. 2012; 16(1):172-7.

6. Cotta RMM, Reis RS, Campos AAO, Gomes AP, Antonio VE, Siqueira-Batista R. Debates atuais em humanização e saúde: quem somos nós. Ciênc Saúde Coletiva. 2013, 18(1):171-9.

7. Valore LA, Ferrarini NL. Escolha e identidade profissional: desafios e possibilidades na formação universitária. Int J Dev Educ Pshyc. 2010, 1(1): 391-8.

8. Magalhães M, Reivo A. Re-opção de curso e maturidade vocacional. Rev ABOP. 1998; 2(2):7-28.

9. Cunha AM, Tunes E, Silva RR. Evasão do curso de química da Universidade de Brasília: a interpretação do aluno evadido. Química Nova. 2001; 24(1):262-80. 
10. Biazus C. Sistema de fatores que levam o aluno a evadir-se dos cursos de graduação na UFSM e na UFSC: um estudo no curso de ciências contábeis. [Tese] Florianópolis (SC): Universidade Federal de Santa Catarina; 2004.

11. Ghizoni LD, Telles MMR. Escolha e reescolha profissional: um estudo sobre estudantes universitários noturnos. In: Lassance MCP, Paradiso AC, Bardagi MP, Sparta M, Frischenbruder SL. Intervenção e compromisso social - Orientação profissional teoria e técnica. São Paulo: Vetor, p. 291-301, 2005.

12. Super DE. Dimensions and measurement of vocational maturity. Teachers College Record, 1955.

13. Super DE. Assessment in career guidance: toward truly developmental counseling. Personnel Guidance J. 1983; 61(9):30-42.

14. Savickas ML. Measuring career development: current status and future directions. Career Develop Quarterly, 1994; 43(1):54-62.

15. Bardagi MP, Boff RM. Autoconceito, autoeficácia profissional e comportamento exploratório em universitários concluintes. Avaliação. 2010; 15(1):41-56.

16. Reul MA et al. Metodologias ativas de ensino aprendizagem na graduação em Odontologia e a contribuição da monitoria-relato de experiência. Rev ABENO, 2016; 16(2):62-8.

17. Mazur E. Peer Instruction: A User's Manual. Prentice Hall, Upper Saddle River, NJ, 1997.

18. Mazur E, Somers MD. Peer instruction: A user's manual. Am J Phys. 1999; 67(4):359400.

19. Crouch $\mathrm{CH}$ et al. Peer instruction: Engaging students one-on-one, all at once. Res Based Reform University Physics. 2007; 1:40-95.

20. Koehler SMF. Inovação didática-projeto de reflexão e aplicação de metodologias ativas de aprendizagem no ensino superior: uma experiência com "peer instruction". Janus. 2012; 9(15):16-26.

21. Mitre SM, Siqueira-Batista R, Girardi-deMendonça JM, Morais-Pinto NM, Meirelles CAB, Pinto-Porto C. Metodologias ativas de ensino-aprendizagem na formação profissional em saúde: debates atuais. Ciênc Saúde Coletiva. 2008;13 Suppl 2: 2133-44.

22. Tsuji H, Aguilar-da-Silva RH. Relato de experiência de um novo modelo curricular: aprendizagem baseada em problemas, implantada na unidade educacional do sistema endocrinológico na $2^{\mathrm{a}}$ série do curso médico da Faculdade de Medicina de Marília Famema. Arq Bras Endocrinol Metab. 2004; 48(4):535-43.

23. Mckenna L, French J. A step ahead: Teaching undergraduate students to be peer teachers. Nurse Educ Pract. 2011; 11(2):141-5.

24. Cezar PHN, Guimarães FT, Gomes AP, Rôças G, Siqueira- Batista R. Transição paradigmática na educação médica: um olhar construtivista dirigido à aprendizagem baseada em problemas. Rev Bras Educ Méd. 2010; 34(2):298-303.

25. Silva KASR. A docência e seus desafios: um olhar crítico acerca da comercialização da educação. Rev Ciênc Hum. 2013, 6(1):107-19.

26. Fernandes Neto, AJ et al. Montagem de modelos de estudo em articulador classe III semi-ajustável - ASA. 2005. [Acesso em 10 de setembro de 2018]. Disponível em: http://www.fo.ufu.br/sites/fo.ufu.br/files/Anex os/Documentos/AnexosRoteiroOclusaoCap13. pdf

\section{Correspondência para:}

Fernanda Nunes de Souza

e-mail: nandasouzanunes@gmail.com

Rua 27 de Janeiro 160, Carambita

27600-000 Valença/RJ 